\title{
Optimal Placement of Readers in an RFID Network Using Particle Swarm Optimization
}

\author{
Indrajit Bhattacharya ${ }^{1}$, Uttam Kumar Roy ${ }^{2}$ \\ 1 Kalyani Govt. Engg. College, Kalyani, Nadia, India, indra51276@gmail.com, \\ 2 Dept. of IT, Jadavpur University, Kolkata-700098, India, u_roy@it.jusl.ac.in
}

\begin{abstract}
An RFID network consists of a set of tags and readers. The cost and the number of tags covered directly depend on the number of readers. So, finding optimal number of readers and their positions to cover all tags is one of the most important issues in an RFID network.

In this paper, we have proposed a reader placement technique in a departmental store equipped with RFID network using Particle Swarm Optimization (PSO). The proposed algorithm finds minimal number of readers along with their position with 100\% coverage of tagged items. Simulated results also show the algorithms effectiveness in achieving the optimal solution.
\end{abstract}

Keywords: RFID, Reader, Tag, PSO, particle, fitness

\section{Introduction}

A departmental store has different types of items. The items span over different racks that are distributed in sections and in multiple floors. In many cases, periodic monitoring and tracking of the items is required. Monitoring the tagged items periodically helps us to gather critical information such as date of expiry, date of entry, physical location (e.g. rack number), rate of sale, volume, cost, stock requirement of the items. RFID [10,4] is the most promising technology for this.

In an RFID-based departmental store, items are tagged with the active/passive RFID tags (hereafter referred to as simply 'tags'), which stores information about the associated items. The RFID readers (hereafter referred to as simply 'readers') are placed in the strategic positions and they form a backbone network. These readers periodically (or upon request) collect information about the tags in its interrogation range and send the information to a central server through the backbone network for further processing.

Since the radio range of the readers is limited to a few meters, typically many readers are required to monitor these items (possibly huge). Hence, for a cost effective implementation of the system, an important issue is to find out the minimal number of readers (along with their positions) that are sufficient enough to cover all the items. The placement of the RFID readers in a RFID network and to find minimal number of readers to cover the entire region is a NP hard problem [15]. Hence suitable optimization algorithm may be applied to solve this problem, which can result in first convergence with minimum resource requirement. The Particle Swarm Optimization (PSO) is a population based optimization technique that requires less amount of computation and with suitable fitness function it can converge to the solution very fast. Hence PSO can be considered as a good choice to solve this type of optimization problem.

This work is partially funded by the UGC PURSE program.

DOI: $10.5121 /$ ijcnc.2010.2615 
In this paper, we have proposed an efficient algorithm, based on Particle Swarm Optimization $[1,2,3,4]$ technique, to find out the minimal number of readers and their positions for maximum coverage of the tagged items placed over different racks of the store.

The RFID technology provides accurate information on the number of items, their locations in the store and their distributions [17, 18, 19], while the proposed algorithm suggests the minimal number of readers (along with their strategic locations) required to completely cover all the items in the store. The proposed algorithm reduces the deployment cost of the network significantly keeping read accuracy of the system unchanged. The proposed algorithm has been implemented in a simulated environment and the experimental results exhibit reasonably good performance.

\section{Related Work}

The problem of providing complete coverage of the items in a departmental store has been addressed using fixed readers. Given a minimum area rectangle (with dimension of XxY) that encloses the area to be covered, the problem of complete coverage using fixed readers is the same as that of covering the entire region of rectangular shape with a number of fixed sized circles, the radius of each circle would be equal to the readers interrogation range, $r$.

In [6], it is shown that maximum coverage that can be achieved with no overlapping among the readers is 0.906899682 . Hence, complete coverage is possible only if there is an overlap among the readers. However, author does not suggest how much overlapping is allowed and how to minimize the number of readers.

In [5], it is stated that the density of an optimal layout, i.e., the ratio of sum total of the area covered by all the circles to the total area to be covered, would be 1.209 . However, the optimal placement of the readers in order to achieve maximum coverage has not been addressed.

It can be observed that only fixed readers may not be cost-effective to cover the entire region as the size of the rectangular region increases. In [7], authors proposed a coverage planning tool for RFID network with mobile readers. The critical issues of this tool are to decide the number of readers required to achieve complete coverage, the placement of the readers and the velocity and movement of the mobile readers. They suggested a scheme for complete coverage of a rectangular area within a specified time period $t$, where $t$ is very small using optimal number of readers.

For many applications such as departmental store, tagged items are placed in respective racks, which are placed only in certain locations in the store. Hence, items can be monitored completely by covering only the racks instead of covering entire region. Moreover, to prevent theft [16], only two additional readers need to be placed at entry and exit point of the store. Apart from that, the readers should be placed in the strategic locations to achieve complete coverage of the items, in an efficient and faster way. The readers are connected via some backbone network which in turn is connected to a central server. Hence, rather than mobile readers, fixed readers approach for achieving complete coverage is sometimes more effective and realistic for most of the applications.

\section{System Description}

The entire departmental store is divided into regions where racks are placed with different orientations, free spaces for passage and the display or billing counters. The tagged items are 
placed on the respective racks characterized by the item types. The prime objective of the system is to periodic monitor of these tagged items using minimal number of readers [15, 20].

Each item that is entered in the store is attached with a tag that contains information of the associated item, which can be described in terms of a record of 5-tuples (TypeOfTheProduct, SubTypeOfTheProduct, Product-id, Position, Date).

The tagging has been done in the following way:

TAG $=$ [TypeOfProduct | Subtype | Product-ID | Position | Date $]$

Each tagged item should have five fields. They are:

a) Type of Products: Such as Garment, Utensils, Food, Electronics goods, etc. Here domain of this field is Alphabets of English language. For example, All Garments products should be tagged by character ' $G$ ' and so on.

b) Subtype of products: For each product type there may be sub product types such as electronics good may be of TV, VCD, etc. The domain of this field is Alphabets of English language.

c) Product-ID: This field denotes a unique number, which is tagged to each product used for identity of the product. The domain of this field is string of 0 's and 1's of 16 bits long.

d) Position: The domain of Position field is simply positive integer. Position field once again sub-categorized into two fields, which are $\mathrm{x}$-coordinate and $\mathrm{y}$-coordinate. According to the Type and Subtype of the item, all items will be stored to their corresponding position (Basically in rack kept in the store).

e) Date: This field is of the pattern dd-mm-yyyy. Whenever an item enters to the store, it is tagged with current date.

After the items are tagged, they are placed to their corresponding position in the rack sequentially according as their Type and Subtype.

\section{Particle Swarm Optimization-A Brief Description}

The Particle Swarm Optimization (PSO) is a new population based optimization technique, which was first proposed by Kennedy and Eberhart in 1995 [1].

The PSO typically begins with a random population and searches for fitness optimum just like the Genetic Algorithm (GA). However, in the PSO algorithm, particles will evolve by cooperation and competition among the individuals themselves through generations instead of using genetic operators [11].

Many variants of PSO [12] have been developed including constrained, multi objective, and discrete or combinatorial versions and applications have been developed using PSO in many fields[13, 14].

Each particle, which is a volume-less entity, flies in the n-dimensional search space. The velocity and position of a particle is adjusted dynamically according to its own flying experience and its companions' flying experience. Each particle keeps track of is coordinates in the problem space, which are associated with the best solution (fitness) it has achieved so far. This value is called pbest. The particle swarm optimizer tracks another best value called gbest which is the global best value obtained so far by any particle in the population.

In an iteration, velocity and position of each particle changes toward its pbest and gbest locations as shown in the following equations: 


$$
\begin{aligned}
& v_{i}^{k+1}=w \cdot v_{i}^{k}+c_{1} r_{1}\left(\text { pbest }_{i}-x_{i}^{k}\right)+c_{2} r_{2}\left(\text { gbest }_{i}-x_{i}^{k}\right) \\
& x_{i}^{k+1}=x_{i}^{k}+v_{i}^{k+1}
\end{aligned}
$$

Here, $x_{i}^{k}$ is current position of particle $\mathrm{i}$ at iteration $\mathrm{k}$, which has velocity $v_{i}^{k}$ and $v_{\min } \leq v_{i}^{k} \leq v_{\max }$ and the pbest $t_{i}$ is the historical best position of $x_{i}^{k}$ and gbest $_{i}$ is the global best position in the population's history. The parameter $\mathrm{w}$ is the inertia weight factor, $\mathrm{c}_{1}$ and $\mathrm{c}_{2}$ are acceleration constants and $r_{1}$ and $r_{2}$ are uniform random numbers between 0 and 1 .

The parameter $v_{\max }$ determines the resolution with which region between the present position and target position is searched. If $v_{\max }$ is too high, particles may fly past the good solutions. If $v_{\max }$ is too small, particles may not explore sufficiently beyond local solutions.

The constants $\mathrm{c}_{1}$ and $\mathrm{c}_{2}$ represent the weighting of the stochastic acceleration terms that pull each particle toward pbest and gbest positions. Low values makes particles to roam far from target regions before being tugged back. On the other hand, high values result in abrupt movements toward, or past, the target regions. It can be proved that for convergence $c_{1}+c_{1}$ must be less than or equal to 4 .

The inertia weight $\mathrm{w}$ provides a balance between global and local exploration and exploitation, and on average results in less iterations required to find a sufficiently good solution. It is typically set according to the following equation:

$w=w_{\max }-\frac{w_{\max }-w_{\min }}{\text { iter }_{\max }} \times$ iter

where iter $_{\max }$ is the maximum iteration number (generation) and iter is the current iteration number.

\section{Implementation}

At first set of candidate RFID readers is considered across the simulation area. The candidate readers denote the possible positions of the readers. For example, Figure 1a) readers may be placed only in sixteen possible positions. The algorithm will finally select (minimal number of) positions, where the readers have to be placed depending upon physical distribution of the items. For example, figure 1b) illustrates that only 4 (four) readers have to be used at positions 3,512 and 15.

The number and positions of candidate readers are selected carefully so that they together can cover the entire region. We have considered candidate positions that are separated by $\sqrt{2} \mathrm{r}$ [Figure 2a)] where $r$ is the read range of the reader. The read accuracy of the readers may be affected by the interference of the wall partitions of the store and racks. The two-ray ground propagation model is considered here. 


\begin{tabular}{|c|c|c|c|c|c|c|}
\hline$\bigcirc_{0}$ & $\bigcirc_{1}$ & $\mathrm{O}_{2}$ & $\mathrm{O}_{3}$ & $\bigcirc_{0}$ & $\bigcirc_{1}$ & $\mathrm{O}_{2}$ \\
\hline $\mathrm{O}_{4}$ & $\bigcirc 5$ & $\mathrm{O}_{6}$ & $\bigcirc_{7}$ & $\bigcirc_{4}$ & 5 & O6 \\
\hline $\mathrm{O}_{8}$ & $\bigcirc 9$ & $\mathrm{O}_{10}$ & $\bigcirc_{11}$ & $\bigcirc 8$ & ○9 & $\mathrm{O}_{10}$ \\
\hline $\mathrm{O}_{12}$ & $\mathrm{O}_{13}$ & $\bigcirc_{14}$ & $\mathrm{O}_{15}$ & $O_{12}$ & $\mathrm{O}_{13}$ & \\
\hline
\end{tabular}

(a)

(b)

Fig. 1. a) Candidate readers in initial phase b) A probable solution (filled circles indicate the positions of the readers that are present).

Since in the final solution, a reader may be present or absent, total number of potential solutions is then $2^{\mathrm{n}}$. Hence, any algorithm that finds out the best solution has the complexity $\mathrm{O}\left(2^{\mathrm{n}}\right)$. In fact, the solution to this problem is NP-Hard [15]. So, it is not a possible to execute this computationally intensive algorithm within a reasonable amount of time as the size of the candidate readers increases.

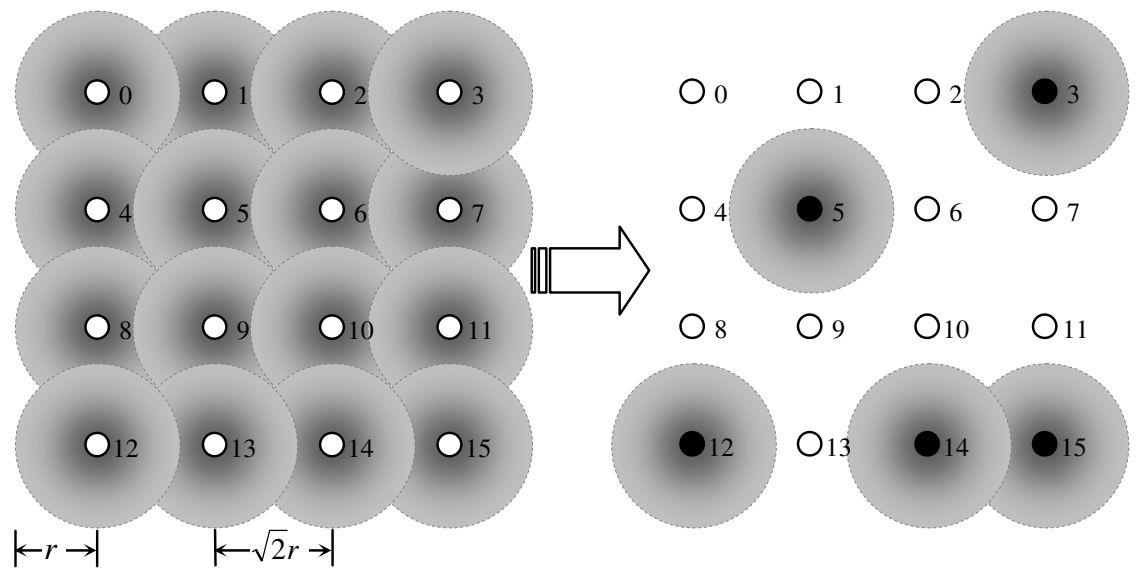

Fig. 2. Overlapping candidate readers b) area coverage of a solution.

The problem representation is one of the most important parameters in the PSO. The particles fly in an $n$-dimensional space where $\mathrm{n}$ is equal to the number of candidate RFID readers. Each particle is a potential solution in PSO. The position of each particle is then a vector of length $\mathrm{n}$. The elements of this position vector position are real numbers as follows:

if position[i] $>0$, reader at the ith position is present.

else the reader at the ith position is absent.

The starting configuration of PSO is created i.e. the particles are initialized with the elements of the position vector set to a value lying between -0.5 to +0.5 .

An example of the position vector of a particle is shown in Figure 3. 


\begin{tabular}{|c|cccccccccccccc|c|c|c|}
0 & 1 & 2 & 3 & 4 & 5 & 6 & 7 & 8 & 9 & 10 & 11 & 12 & 13 & 14 & 15 \\
\hline-0.23 & -0.32 & -0.19 & 0.45 & -0.45 & 0.12 & -0.36 & -0.25 & -0.15 & -0.24 & -0.36 & -0.14 & 0.32 & -0.28 & 0.46 & 0.13 \\
\hline
\end{tabular}

Fig. 3. The position of a particle corresponding the to the solution Figure 1a).

\subsection{Placement methodology}

Figure 4 illustrates overall reader placement methodology.

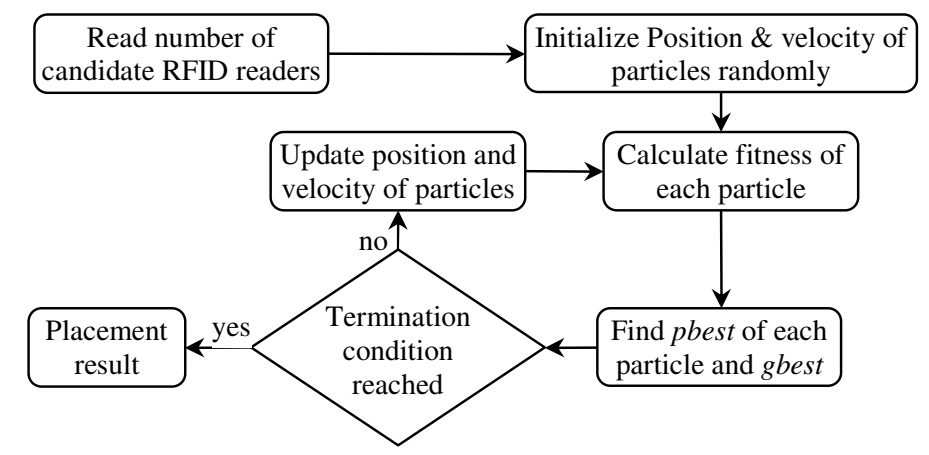

Fig. 4. RFID reader placement methodology.

In the beginning, positions and velocities of the particles are randomly generated. In each generation (iteration), fitness of each particle is evaluated and pbest and gbest are determined. Then position and velocity of each particle is updated using (1) and (4) respectively. This process is repeated until termination condition is met. Termination conditions are generation number limit, run-time limit, or convergence of algorithm to a predefined fitness value. We have used generation number limit as the termination condition.

At any time, the fittest particle in a generation is one that represents the best solution obtained so far. The position vector of the fittest particle determines which readers are really present. For example, if figure 2 shows the position vector of the fittest particle, then the corresponding solution is shown in figure 1a). Since the $0^{\text {th }}$ element - ve, no reader has to be placed at position 0 . Similarly, since $3^{\text {rd }}$ element is +ve, a reader is has to be placed at position 3 .

\subsection{Fitness Function}

In order to evaluate a solution, we need to know the goodness (fitness) of the solution; so we require some metrics. Usually a set of metrics are combined and a fitness function is obtained that guides the optimization process.

We have considered the following matrices i) number of items covered ii) number of RFID readers placed and iii) amount of overlapped area. Since the main objective of the algorithm is minimizing the number of readers while maintaining the $100 \%$ item coverage, given the following parameters,

$\mathrm{c}=$ total items covered

$n=$ number of possible readers that can be placed over the entire region (a subset

of those readers constitute the final solution)

$e=$ points covered by multiple RFID readers

$T=$ total items present

$N=$ no of candidate readers; 


$$
\begin{array}{ll}
X=\frac{c}{T} & {[0 \leq X \leq 1 \text { and } X \text { is alinearlincresing functionf } c]} \\
Y=\frac{N-n}{N} & {[0 \leq Y \leq 1 \text { and } Y \text { is a linearly decrea } \sin g \text { function of } n]} \\
Z=\frac{T-e}{T} & {[0 \leq Z \leq 1 \text { and } Z \text { is a linearly decrea } \sin g \text { function of } e]}
\end{array}
$$

the fitness of a particle is calculated as follows:

fitness $($ position of a particle $)=0.6 * X+0.2 * Y+0.2 * Z$

Since area coverage is the most important factor, the associated weight is chosen larger compared to other weights.

\section{Simulation Test-bed}

In the PSO implementation we have considered a problem space, which is the area covered by the departmental store. We have carried out our simulation in a 500x300 pixel area. A set of rectangular artificial racks are then placed at different positions. We have assumed that items can be placed on at pixel within the racks only. The transmission range of readers and tags is set to 35 pixels wide. The candidate positions of the readers are then separated by $50(\approx \sqrt{ } 2 \times 35)$ pixels. There is one entry and one exit point in the store. The particles are entered to the store through the particular entry point and then the actual tagging is done. In the tagging procedure we have considered the five attributes for each item. They are the product type, subtype, product-id, (initial $x$-coordinate, initial $y$-coordinate), and the date of entry. The unique attribute of an item is the product-id. The items are place in the racks and will obtain its position (x,y) depending upon it's type and subtype.

The RFID readers would be placed in the strategic locations of the store in order to guarantee the maximum coverage of the system with an increased read accuracy. The read accuracy of the readers may be affected by the interference of the wall partitions of the store along with the racks. Since walls of the racks and store attenuate the signal strength, we have used two-way ground propagation model. Initially it is considered that the readers are placed at distance of $\sqrt{ } 2 \mathrm{r}$ apart for allowing overlap among the read range of the neighboring readers where $r$ is the read range of the reader.

From this initial distribution a number of readers are arbitrary chosen as the initial configuration for PSO. This choice depends on the number of bits required to represent a particle in PSO. The PSO algorithm is then applied with the appropriate fitness function which can decide after a number of iteration the fittest particle which gives the desired combination of readers that need to be kept on in the system in order to achieve the maximum coverage with redundant readers has been eliminated.

\section{Simulation Results}

The results after 10,000 iterations are shown in the Figure 5. Figure 5a) shows the positions of the candidate readers. Figure $5 \mathrm{~b}$ ) shows the readers selected after 10,000 iterations. It may be noted that the number of access points is minimized and their positions are selected by the PSO. The effective signal coverage of the selected readers is shown in Figure 5c) and 5d) where black indicates maximum signal strength and white indicates 0 signal strength. Here the signal strength of a reader attenuates by the obstructions such as walls of the racks and store partitions. 
Table 1. PSO parameters used.

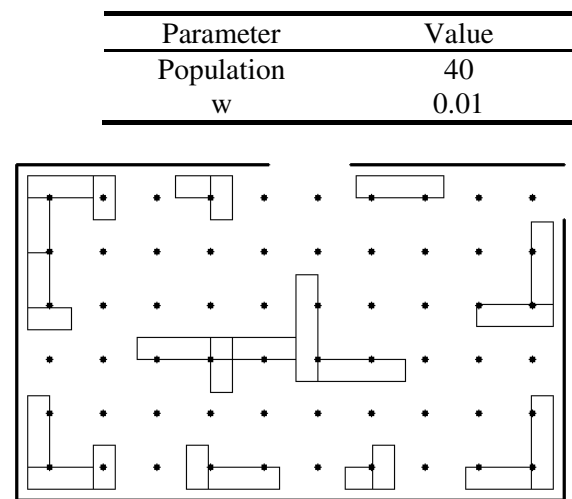

(a)

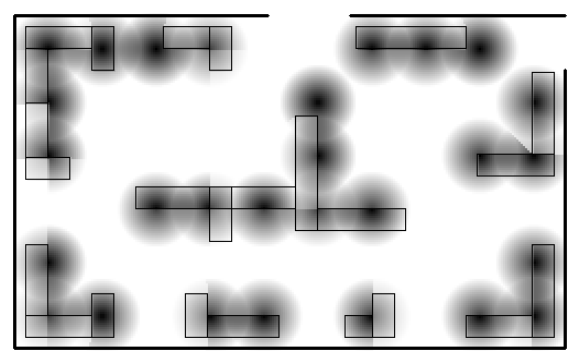

(c)

\begin{tabular}{cc} 
Parameter & Value \\
\hline $\mathrm{c} 1$ & 0.1 \\
$\mathrm{c} 2$ & 0.1 \\
\hline
\end{tabular}

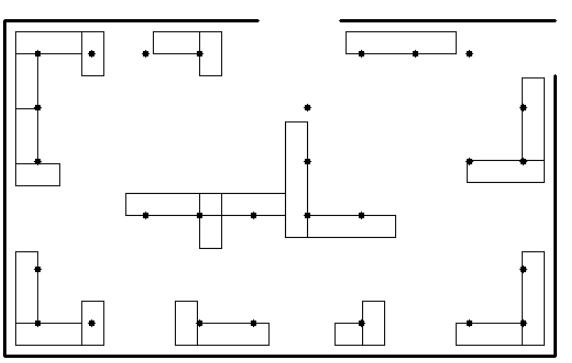

(b)

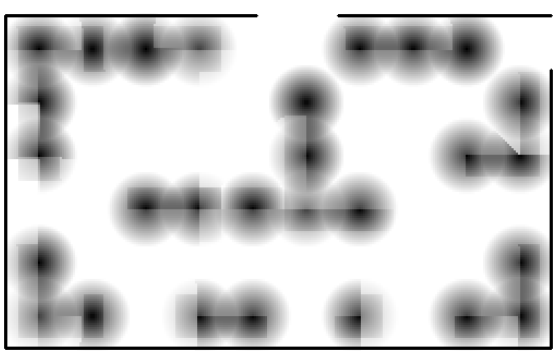

(d)

Fig. 5. Simulation results. a) candidate reader positions b) readers selected c) signal mapping (racks shown) d) signal mapping (racks not shown)

It can be observed the selected readers after applying the PSO algorithm can preserve complete coverage of the items kept in the racks of the store with redundant readers eliminated. Along with that the overlapping areas among the neighboring readers has been minimized by the algorithm.

Table 2. PSO results.

\begin{tabular}{ccc}
\hline No. on iteration & $\%$ of items covered & No. of RFID readers selected \\
\hline 1000 & 95 & 35 \\
10,000 & 100 & 28 \\
100,000 & 100 & 28 \\
\hline
\end{tabular}

\section{Conclusion}

In this paper the problem of systematic periodic monitoring of the items in a departmental store has been addressed so that the items can be monitored with minimum number of readers placed in the strategic locations of the store. It should be noted that for this type of applications rather than monitoring the entire store it is typically required to monitor only the tagged items placed at the different racks in the store. Applying PSO algorithm in the application it has been possible to identify the targeted readers that should be kept on from an initially placed array of readers with the redundant readers being eliminated. The success of the algorithm depends on the choice of the fitness function while implementing the PSO algorithm. In this implementation 
the fitness function has been chosen on three factors as described in the earlier section and has been proved to be working well with the successful demonstration of the simulated results.

\section{References}

1. J. Kennedy and R. Eberhart, "Particle swarm optimization," in Proc. IEEE Intl. Conf. Neural Networks, vol. 4, 1995, pp. 1942-1948.

2. J.Kennedy "The Particle swarm: social adaptation of knowledge" Proc. IEEE International Conference on Evolutionary Computation (Indianapolis, Indiana), IEEE Service Center, Piscataway, NJ, 303-308.

3. P.J.Angeline "Using selection to improve particle swarm optimization" IEEE International Conference on Evolutionary Computation, Anchorage, Alaska, May 4-9, 1998.

4. Qiang Guan, Yu Liu, Yiping Yang, Wesshueg Yu "Genetic Approach for Network Planning in RFID Systems" Proceedings of the sixth International Conference on Intelligent Systems (ISDA '06).

5. R. Kershner "The number of circles covering a set "American Journal of Mathematics, vol. 61, 665671 (1939)

6. S. Anusha "RFIDcover - a coverage planning tool for RFID networks with mobile readers. Masters thesis, Indian Institute of Technology Bombay (2005).

7. S. Anusha, Sridhar Iyer "RFIDcover - A Coverage Planning Tool for RFID Networks with Mobile Readers" Lecture Notes in Computer Science, Embedded and Ubiquitous Computing, Vol. 3823 pages 1047-1057, 2005

8. Wail Gueaieb and Md. Suruz Miah "Mobile Robot Navigation using PSO and Noisy RFID communication" IEEE International Conference on Computational Intelligence for Measurement Systems and Applications, 14-16 July, 2008.

9. Y.H.Shi, R.C. Eberhart "A modified Particle swarm optimizer. IEEE International Conference on Evolutionary Computation” Anchorage, Alaska, May 4-9, 1998.

10. Y.M. Siu, W.S.Chan, K.K. Soo, C.W.Leung, S.M. Law "Exhibition Security and Safety System Using RFID Technology and a modified Particle Swarm optimization Algorithm"

11. Y. Shi and R. Eberhart, "A modified particle swarm optimizer," in Proc. IEEE World Cong. on Computational Intelligence, 1998, pp. 96-73.

12. R. Poli "Analysis of the publications on the applications of particle swarm optimization" Journal of Artificial Evolution and applications, Article ID 685175, 10 pages, 2008.

13. M.S. Rodrigo, Carlos L.S.S. Sobrinho, S.Araujo, Farias Rubem "Particle Swarm Optimization Applied for Locating an Intruder by an Ultra-Wideband Radar Network" Federal University of Para, Brazil

14. Hui Zhu, Syahrulanuar Ngah, Ying Xu, Yuji Tanabe, Takaaki Baba "A Random Time-Varying PSO for local positioning systems," IJCSNS, Vol 8. No. 6, June, 2008.

15. B.Carbunar, M.K.Ramanathant, M.Koyuturk, C.Hoffmannt, and A.Grama, "Redundant reader elimination in RFID systems," in Proceeding of the $2^{\text {nd }}$ Annual IEEE Communications Society Conference on Sensor and Ad hoc Communications and Networks (SECON '05), pp. 176-184, Santa Clara, USA, September 2005.

16. A.Juels, R.L.Rivest, and M.Szydlo, "The blocker tag: selective blocking of RFID tags for consumer privacy," in Proceedings of the $10^{\text {th }}$ ACM Conference on Computer and Communications Security (CCS '03), pp 103-111, Washington, DC, USA, October 2003.

17. D.W.Engels and S.E.Sarma, "The reader collision problem," in Proceeding of the IEEE International Conference on Systems, Man and Cybernetics (SMC '02), vol. 3, pp. 641-646, Hammamet, October 2002.

18. J.Waldrop, D.W.Engels, and S.E.Sarma, "Colorwave: an anticollision algorithm for the reader collision problem," in Proceeding of the International Conference on Communications (ICC'03), vol. 2, pp.1206-1210, Anchorage, Alaska, USA, May 2003.

19. Y.Tanaka and I.Sasase, "Interference avoidance algorithms for passive FRID systems using contention-based transmit abortion," IEICE Transactions on Communications, vol. E90-B, no. 11, pp. 3170-3180, 2007. 
International Journal of Computer Networks \& Communications (IJCNC) Vol.2, No.6, November 2010

20. B.Carbunar, A.Grama, J.Vitek, and O.Carbunar, "Redundancy and coverage detection in sensor networks," ACM Transactions on Sensor Networks, vol. 2, no. 1, pp. 94-128, 2006.

Indrajit Bhattacharya has done his M.Tech in Computer Science \& Engineering from University of Calcutta, India in the year 2002 and M.Sc. from the same University in the year 2000. He is currently associated with Kalyani Govt. Engineering College, Kalyani, India as Assistant Professor in Department of Computer Applications. His research interest lies in the area of Mobile Adhoc network, Wireless Sensor Network and RFID systems.

Dr. Uttam Kumar Roy has got his $\mathrm{PhD}$ degree in Engineering from the Jadavpur University, Kolkata. He has done his M.E in Computer Science \& Engineering from Jadavpur University, India in the year 2002 and B.Tech from the University of Calcutta, India in the year 2000. He is currently associated with Jadavpur University, Kolkata, India as Assistant Professor in Department of IT. His research interest lies in the area of Wireless Sensor Network, Service Oriented Architecture and Distributed Computing.
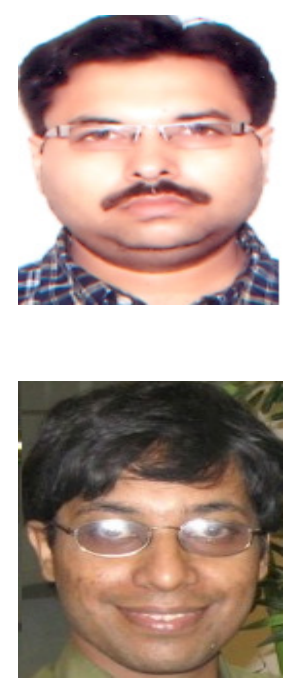